Proceedings of the Edinburgh Mathematical Society (2008) 51, 495-508 (C)

DOI:10.1017/S0013091506000988 Printed in the United Kingdom

\title{
GLOBAL ATTRACTIVITY IN A PREDATOR-PREY SYSTEM WITH PURE DELAYS
}

\author{
X. H. TANG ${ }^{1}$ AND XINGFU ZOU ${ }^{2}$ \\ ${ }^{1}$ Department of Applied Mathematics, Central South University, Changsha, \\ Hunan 410083, People's Republic of China (tangxh@mail.csu.edu.cn) \\ ${ }^{2}$ Department of Applied Mathematics, University of Western Ontario, \\ London, Ontario N6A 5B7, Canada (xzou@uwo.ca)
}

(Received 18 August 2006)

\begin{abstract}
We consider a delay predator-prey system without instantaneous negative feedback and establish some conditions for global attractivity of the positive equilibrium of the system which generalize and improve some of the existing ones. When the system is decoupled, one of the main results reduces to the well-known Wright $3 / 2$ stability condition for the delayed logistic equation.
\end{abstract}

Keywords: predator-prey system; pure delay; global attractivity

2000 Mathematics subject classification: Primary 34K20; 90D25

\section{Introduction}

We consider the global attractivity of the positive equilibrium of a predator-prey system with delays modelled by

$$
\left.\begin{array}{l}
\dot{N}_{1}(t)=N_{1}(t)\left[a_{1}-b_{1} N_{1}\left(t-\tau_{1}\right)-c_{1} N_{2}\left(t-\sigma_{1}\right)\right], \\
\dot{N}_{2}(t)=N_{2}(t)\left[-a_{2}+c_{2} N_{1}\left(t-\sigma_{2}\right)-b_{2} N_{2}\left(t-\tau_{2}\right)\right],
\end{array}\right\}
$$

with initial conditions

$$
N_{i}(t)=\phi_{i}(t) \geqslant 0, \quad t \in[-\Delta, 0], \quad \phi_{i}(0)>0, \quad i=1,2,
$$

where $a_{i}, b_{i}>0, c_{i} \geqslant 0, \tau_{i}, \sigma_{i} \geqslant 0$ for $i=1,2$ and $\Delta=\max \left\{\tau_{1}, \tau_{2}, \sigma_{1}, \sigma_{2}\right\}$.

When the predator species is absent, the prey species is governed by the well-known delay logistic equation

$$
\left.\begin{array}{c}
\dot{N}_{1}(t)=N_{1}(t)\left[a_{1}-b_{1} N_{1}\left(t-\tau_{1}\right)\right], \\
N(s) \geqslant 0 \quad \text { for } s \in\left[-\tau_{1}, 0\right], \quad N(0)>0 .
\end{array}\right\}
$$

For (1.3), a well-known result (usually referred as Wright's $3 / 2$ criterion) is that if $a_{1} \tau_{1} \leqslant$ $\frac{3}{2}$, then the positive equilibrium $a_{1} / b_{1}$ is globally attractive. 
On the other hand, if all delays are zero in (1.1), then system (1.1) simplifies to the following autonomous system of ordinary differential equations:

$$
\left.\begin{array}{l}
\dot{N}_{1}(t)=N_{1}(t)\left[a_{1}-b_{1} N_{1}(t)-c_{1} N_{2}(t)\right], \\
\dot{N}_{2}(t)=N_{2}(t)\left[-a_{2}+c_{2} N_{1}(t)-b_{2} N_{2}(t)\right] .
\end{array}\right\}
$$

It is well known that all positive solutions $N(t)=\left(N_{1}(t), N_{2}(t)\right)$ of (1.4) satisfy $N(t) \rightarrow$ $N^{*}=\left(N_{1}^{*}, N_{2}^{*}\right)$ as $t \rightarrow \infty$ if and only if

$$
a_{1} c_{2}-a_{2} b_{1}>0
$$

where

$$
N_{1}^{*}=\frac{a_{1} b_{2}+a_{2} c_{1}}{b_{1} b_{2}+c_{1} c_{2}}, \quad N_{2}^{*}=\frac{a_{1} c_{2}-a_{2} b_{1}}{b_{1} b_{2}+c_{1} c_{2}} .
$$

From this fundamental result, one naturally expects that under (A1) $N^{*}$ remains globally attractive for (1.1), (1.2) if the delays are sufficiently small. This expectation was confirmed recently by $\mathrm{He}[\mathbf{7}]$. Indeed, by constructing a Lyapunov functional, $\mathrm{He}$ established the following theorem.

Theorem 1.1. Suppose that (A1) holds. Then the positive equilibrium $N^{*}$ for (1.1) is globally attractive, provided that

$$
\left(1+M_{1}^{2}\right) \tau_{1}+\frac{c_{1}}{b_{2}}\left\{\tau_{1}+\left[1+\frac{c_{2}}{b_{1}}\left(1+M_{2}^{2}\right)\right] \sigma_{1}+M_{1}^{2} \sigma_{2}+M_{2}^{2} \tau_{2}\right\}<2,
$$

and

$$
\left(1+M_{2}^{2}\right) \tau_{2}+\frac{c_{2}}{b_{1}}\left\{\tau_{2}+\left[1+\frac{c_{1}}{b_{2}}\left(1+M_{1}^{2}\right)\right] \sigma_{2}+M_{1}^{2} \tau_{1}+M_{2}^{2} \sigma_{1}\right\}<2
$$

where

$$
M_{1}=\frac{a_{1}}{b_{1}} \mathrm{e}^{a_{1} \tau_{1}} \quad \text { and } \quad M_{2}=\frac{-a_{2}+c_{2} M_{1}}{b_{2}} \mathrm{e}^{\left(-a_{2}+c_{2} M_{1}\right) \tau_{2}} .
$$

Hofbauer and So [9] studied a general Lotka-Volterra system allowing distributed delays but with instantaneous negative feedback, which, in the case of $n=2$ and in the context of predator-prey, includes the following system:

$$
\left.\begin{array}{l}
\dot{N}_{1}(t)=N_{1}(t)\left[a_{1}-b_{1} N_{1}(t)-c_{1} N_{2}\left(t-\sigma_{1}\right)\right], \\
\dot{N}_{2}(t)=N_{2}(t)\left[-a_{2}+c_{2} N_{1}\left(t-\sigma_{2}\right)-b_{2} N_{2}(t)\right],
\end{array}\right\}
$$

The main theorem of [9] can be stated, as below, in terms of (1.9).

Theorem 1.2. Suppose that (A1) holds. Then the positive equilibrium $N^{*}$ for (1.9) with (1.2) is globally attractive for all $\sigma_{1}$ and $\sigma_{2}$ if and only if $b_{1} b_{2}-c_{1} c_{2}=0$ or

$$
b_{1} b_{2}-c_{1} c_{2}>0
$$


Theorem 1.2 is proved by constructing a Lyapunov functional, taking advantage of the fact that there is no delay in the negative feedback terms $b_{1} N_{1}(t)$ and $b_{2} N_{2}(t)$ (i.e. the system has instantaneous negative feedbacks).

From Theorem 1.2, we see that under the diagonal dominating condition (DD), the off-diagonal delays do not affect the global attractivity of $N^{*}$ (assuming (A1)). This suggests that one only needs to worry about the diagonal delays in this context. He [6] made an attempt to partly address this problem by considering one diagonal delay. In fact, He considered the system

$$
\left.\begin{array}{l}
\dot{N}_{1}(t)=N_{1}(t)\left[a_{1}-b_{1} N_{1}(t-\tau)-c_{1} N_{2}\left(t-\sigma_{1}\right)\right] \\
\dot{N}_{2}(t)=N_{2}(t)\left[-a_{2}+c_{2} N_{1}\left(t-\sigma_{2}\right)-b_{2} N_{2}(t)\right]
\end{array}\right\}
$$

and established the following result.

Theorem 1.3. Assume that (A1) holds, and that

$$
\frac{c_{1} c_{2}}{b_{1} b_{2}}<\frac{1-a_{1} \tau \mathrm{e}^{a_{1} \tau}}{1+a_{1} \tau \mathrm{e}^{a_{1} \tau}}
$$

Then the equilibrium $N^{*}$ is globally attractive for (1.10) with $\sigma_{1}=\sigma_{2}$.

Obviously, (1.11) implies (DD). Note that (1.11) is equivalent to

$$
a_{1} \tau \mathrm{e}^{a_{1} \tau}<\frac{b_{1} b_{2}-c_{1} c_{2}}{b_{1} b_{2}+c_{1} c_{2}}
$$

which coincides with (DD) when $\tau=0$. Therefore, under (DD), (1.12) gives an estimate for the smallness of $\tau$ with which $N^{*}$ remains globally attractive for (1.10) with $\sigma_{1}=\sigma_{2}$.

Observe that if the capture rate $c_{1}=0$, the prey species again is governed by (1.3). As mentioned before, when $a_{1} \tau_{1} \leqslant \frac{3}{2}$, every positive solution $N_{1}(t)$ of $(1.3)$ tends to $N_{1}^{*}=a_{1} / b_{1}$, and thus the equation for $N_{2}$ in (1.1) can be considered as an asymptotically autonomous equation with the limiting equation

$$
\dot{N}_{2}(t)=N_{2}(t)\left[a-b_{2} N_{2}\left(t-\tau_{2}\right)\right]
$$

where $a=-a_{2}+\left(c_{2} a_{1}\right) / b_{1}>0$ under (A1). By the theory of asymptotically autonomous systems (see, for example, [2]) and Wright's criterion, one knows that the $N_{2}$ component of the solution of (1.1) converges to $N_{2}^{*}$ as $t \rightarrow \infty$, provided that $a \tau_{2} \leqslant \frac{3}{2}$, which holds when $\tau_{2}=0$.

With the above observation in mind, we feel that Theorems 1.1-1.3 are not satisfactory at least in the following sense. The restrictions (1.6) and (1.7) in Theorem 1.1 and (1.11) in Theorem 1.3 for smallness of delays do not reduce to Wright's $3 / 2$ criterion when the system (1.1) is decoupled by letting $c_{1}=0$. Moreover, Theorem 1.3 was only for a special case of (1.1) (i.e. $\tau_{2}=0$ and $\sigma_{1}=\sigma_{2}$ ), and even in such a special case, as observed above, (1.12) can be improved.

Motivated by the above dissatisfaction, and encouraged by the authors' recent work $[\mathbf{2 7}, \mathbf{2 9}]$, where 3/2-type criteria were obtained for the delayed competitive system of 
Lotka-Volterra type without instantaneous negative feedback, we will establish some criteria of $3 / 2$ type for the global attractivity of the positive equilibrium $N^{*}$. Note that, owing to the lack of instantaneous negative feedback, the global attractivity of systems 'without instantaneous negative feedback' (or 'of pure-delay type') becomes much more difficult and has been studied by Gopalsamy [3], Gopalsamy and He [5], He [6-8], Kuang $[\mathbf{1 2}, \mathbf{1 3}]$, Kuang and Smith $[\mathbf{1 4}, \mathbf{1 5}]$, Smith $[\mathbf{1 9}]$, So et al. $[\mathbf{2 3}]$ and Tang and Zou $[\mathbf{2 8}]$. Also note that $3 / 2$-type stability criteria for various scalar delay-differential equations are available in $[\mathbf{1}, \mathbf{1 0}-\mathbf{1 2}, \mathbf{1 6}-\mathbf{1 8}, \mathbf{2 0}-\mathbf{2 2}, \mathbf{2 4}-\mathbf{2 6}, \mathbf{3 1}-\mathbf{3 4}]$.

The rest of the paper is organized as follows. In $\S 2$, we give the main results. In $\S 3$, we establish some preliminary lemmas, which address the persistence and dissipativity of system (1.8) and therefore, are of some interest and importance themselves. In $\S 4$, by combing these lemmas with the 'sandwiching' technique and using some subtle techniques of integration and inequality, we give the proofs of the main theorems.

\section{Main results}

Theorem 2.1. Assume that (A1) and (DD) hold, and that

$$
b_{1}\left(a_{1} b_{2}+a_{2} c_{1}\right) \tau \leqslant \frac{3}{2}\left(b_{1} b_{2}-c_{1} c_{2}\right)+\frac{c_{1} c_{2}\left(b_{1} b_{2}-c_{1} c_{2}\right)}{2\left(b_{1} b_{2}+c_{1} c_{2}\right)} .
$$

Then the positive equilibrium $N^{*}=\left(N_{1}^{*}, N_{2}^{*}\right)$ of (1.10) is a global attractor.

It is easily seen that, by letting $c_{1}=0$, Theorem 2.1 reproduces Wright's $3 / 2$ result for the autonomous delayed logistic equation (1.3). Note that the above $3 / 2$-type condition (2.1) is established for (1.10), where only one diagonal delay is present. In the case when both diagonal delays are present, i.e. system (1.1), we are unable to obtain a similar result by our method. The main difficulty is that in the case $\tau_{2} \neq 0$ we cannot determine the two important inequalities (4.6) and (4.7) from (4.4), but these play a key role in the proof of Theorem 2.1. However, the following theorem allows small $\tau_{2}>0$, which is along the lines of Theorem 1.2.

Theorem 2.2. Let

$$
\begin{aligned}
& M_{1}=\frac{a_{1}}{b_{1}} \exp \left(a_{1} \tau_{1}+\mathrm{e}^{-a_{1} \tau_{1}}-1\right), \\
& M_{2}=\frac{-a_{2}+c_{2} M_{1}}{b_{2}} \exp \left[\left(-a_{2}+c_{2} M_{1}\right) \tau_{2}+\mathrm{e}^{-\left(-a_{2}+c_{2} M_{1}\right) \tau_{2}}-1\right],
\end{aligned}
$$

and

$$
B_{i}= \begin{cases}\frac{\left[2-\left(M_{i} b_{i} \tau_{i}\right)^{2}\right]}{\left[2+\left(M_{i} b_{i} \tau_{i}\right)^{2}\right]} & \text { if } M_{i} b_{i} \tau_{i} \leqslant 1, \\ \frac{\left[3-2\left(M_{i} b_{i} \tau_{i}\right)\right]}{\left[1+2\left(M_{i} b_{i} \tau_{i}\right)\right]} & \text { if } M_{i} b_{i} \tau_{i}>1\end{cases}
$$

for $i=1,2$. Assume that (A1) and (DD) hold, and that

$$
\frac{c_{1} c_{2}}{b_{1} b_{2}}<B_{1} B_{2} \text {. }
$$

Then the positive equilibrium $N^{*}$ for (1.1) is a global attractor. 
When $\tau_{2}=0, B_{2}=1$, and we thereby have the following result for $(1.10)$.

Corollary 2.3. Assume that $(A 1)$ and $(D D)$ hold and that

$$
\frac{c_{1} c_{2}}{b_{1} b_{2}}< \begin{cases}\frac{\left[2-\left(\tau M_{1} b_{1}\right)^{2}\right]}{\left[2+\left(\tau M_{1} b_{1}\right)^{2}\right]} & \text { if } \tau M_{1} b_{1} \leqslant 1 \\ \frac{\left[3-2\left(\tau M_{1} b_{1}\right)\right]}{\left[1+2\left(\tau M_{1} b_{1}\right)\right]} & \text { if } \tau M_{1} b_{1}>1,\end{cases}
$$

where $M_{1}$ is defined by (2.2). Then the positive equilibrium $N^{*}$ for (1.10) is a global attractor.

Remark 2.4. In view of the proof of [7, Theorem 1.1], if the $M_{1}$ and $M_{2}$ in (1.6) and (1.7) are replaced by (2.2) and (2.3), respectively, the conclusion in Theorem 1.1 still holds.

Remark 2.5. Theorems 2.2 and 1.1 are complementary. In Theorem 1.1 , the condition (DD) on the coefficients of (1.1) is not needed, but the restrictions on the off-diagonal delays are added, whereas Theorem 2.2 is contrary to Theorem 1.1.

Remark 2.6. When $\tau_{1}=\tau_{2}=0, B_{1}=B_{2}=0$ and (2.5) reduces to (DD). Thus, in such a special case of $n=2$ and in the predator-prey context, Theorem 1.2 is slightly less restrictive than Theorem 2.2, with the difference being between the use of 'nonnegative' and 'positive' for the term $b_{1} b_{2}-c_{1} c_{2}$. However, as stated in the title and in $\S 1$, dealing with positive diagonal delays $\tau_{1}$ and $\tau_{2}$ is the primary goal of this work, which Theorem 1.2 fails to acheive.

Remark 2.7. In condition (2.6),

$$
\tau M_{1} b_{1}=a_{1} \tau \exp \left(a_{1} \tau+\mathrm{e}^{-a_{1} \tau}-1\right)<a_{1} \tau \mathrm{e}^{\left(a_{1} \tau\right)^{2} / 2}
$$

Hence, condition (2.6) improves on (1.11) greatly.

\section{Preliminary lemmas}

In this section, we give some lemmas which will be used in $\S 4$ in the proofs of the main theorems. The first one is from [29].

Lemma 3.1. Let $a>0$ and $0<\mu<1$. Then the system of inequalities

$$
\left.\begin{array}{l}
y \leqslant(a+\mu x) \exp \left[\frac{1-\mu}{a} x-\frac{(1-\mu)^{2}(1+2 \mu)}{6 a^{2}(1+\mu)} x^{2}\right]-a, \\
x \leqslant a-(a-\mu y) \exp \left[-\frac{1-\mu}{a} y-\frac{(1-\mu)^{2}(1+2 \mu)}{6 a^{2}(1+\mu)} y^{2}\right]
\end{array}\right\}
$$

has a unique solution: $(x, y)=(0,0)$ in the region $D=\{(x, y): 0 \leqslant x<a, 0 \leqslant y<a / \mu\}$. 
Lemma 3.2. Assume that (A1) holds and let $\left(N_{1}(t), N_{2}(t)\right)$ be the solution of (1.1) and (1.2). Then eventually

$$
0<N_{i}(t) \leqslant M_{i}, \quad i=1,2,
$$

where $M_{1}$ and $M_{2}$ are defined by (2.2) and (2.3), respectively.

Proof. From (1.1) and (1.2), it is easy to see that $N_{i}(t)>0$ for $t \geqslant 0$ and $i=1,2$. Hence,

$$
\dot{N}_{1}(t) \leqslant N_{1}(t)\left[a_{1}-b_{1} N_{1}\left(t-\tau_{1}\right)\right] \leqslant a_{1} N_{1}(t), \quad t \geqslant 0 .
$$

If $N_{1}(t) \leqslant a_{1} / b_{1}$ eventually, then the first inequality in (3.2) holds naturally for large $t$ and $i=1$. If $N_{1}(t) \geqslant a_{1} / b_{1}$ eventually, then it follows from (3.3) that $\lim _{t \rightarrow \infty} N_{1}(t)=a_{1} / b_{1}$, and so (3.2) holds for large $t$ and $i=1$. In what follows, we consider only the case when $N_{1}(t)$ oscillates on $a_{1} / b_{1}$. Let $t^{*}$ be an arbitrary local left maximum point of $N_{1}(t)$ such that $N_{1}\left(t^{*}\right)>a_{1} / b_{1}$. Then $\dot{N}_{1}\left(t^{*}\right) \geqslant 0$, and it follows from (3.3) that there exists $\xi \in\left[t^{*}-\tau_{1}, t^{*}\right]$ such that $N_{1}(\xi)=a_{1} / b_{1}$. For $t \in\left[\xi, t^{*}\right]$, integrating (3.3) from $t-\tau_{1}$ to $\xi$, we get

$$
-\ln \frac{N_{1}\left(t-\tau_{1}\right)}{N_{1}(\xi)} \leqslant a_{1}\left(\xi+\tau_{1}-t\right), \quad \xi \leqslant t \leqslant t^{*}
$$

Thus,

$$
N_{1}\left(t-\tau_{1}\right) \geqslant \frac{a_{1}}{b_{1}} \exp \left[-a_{1}\left(\xi+\tau_{1}-t\right)\right], \quad \xi \leqslant t \leqslant t^{*} .
$$

Substituting this into the first inequality in (3.3), we obtain

$$
\frac{\dot{N}_{1}(t)}{N_{1}(t)} \leqslant a_{1}\left\{1-\exp \left[-a_{1}\left(\xi+\tau_{1}-t\right)\right]\right\}, \quad \xi \leqslant t \leqslant t^{*} .
$$

Integrating (3.4) from $\xi$ to $t^{*}$, we have

$$
\begin{aligned}
\ln \frac{b_{1} N_{1}\left(t^{*}\right)}{a_{1}} & \leqslant a_{1} \int_{\xi}^{t^{*}}\left\{1-\exp \left[-a_{1}\left(\xi+\tau_{1}-t\right)\right]\right\} \mathrm{d} t \\
& =a_{1}\left(t^{*}-\xi\right)+\mathrm{e}^{-a_{1} \tau_{1}}-\exp \left[-a_{1}\left(\xi+\tau_{1}-t^{*}\right)\right] \\
& =a_{1}\left(t^{*}-\xi\right)-\exp \left[-a_{1}\left(\xi+\tau_{1}-t^{*}\right)\right]+\mathrm{e}^{-a_{1} \tau_{1}} \\
& \leqslant a_{1} \tau_{1}-1+\mathrm{e}^{-a_{1} \tau_{1}} .
\end{aligned}
$$

Here we have used the fact that the function $f(x)=x-\mathrm{e}^{x-a \tau_{1}}$ is increasing in the interval $\left[0, a \tau_{1}\right]$ and hence $f(x) \leqslant f\left(a \tau_{1}\right)=a \tau_{1}-1$ for $x \in\left[0, a \tau_{1}\right]$. The above inequality implies that

$$
N_{1}\left(t^{*}\right) \leqslant \frac{a_{1}}{b_{1}} \exp \left(a_{1} \tau_{1}+\mathrm{e}^{-a_{1} \tau_{1}}-1\right)
$$

It follows that, for large $t$,

$$
N_{1}(t) \leqslant \frac{a_{1}}{b_{1}} \exp \left(a_{1} \tau_{1}+\mathrm{e}^{-a_{1} \tau_{1}}-1\right)=M_{1}
$$


Choose $T>0$ such that $N_{1}(t) \leqslant M_{1}$ for $t \geqslant T$. Then from (1.1), we have

$$
\dot{N}_{2}(t) \leqslant N_{2}(t)\left[-a_{2}+c_{2} M_{1}-b_{2} N_{2}\left(t-\tau_{2}\right)\right], \quad t \geqslant T+\Delta .
$$

Note that

$$
c_{2} M_{1}-a_{2}=c_{2} \frac{a_{1}}{b_{1}} \exp \left(a_{1} \tau_{1}+\mathrm{e}^{-a_{1} \tau_{1}}-1\right)-a_{2}>\frac{a_{1} c_{2}-a_{2} b_{1}}{b_{1}}>0 .
$$

Hence, similarly, from (3.5) we eventually have

$$
N_{2}(t) \leqslant \frac{-a_{2}+c_{2} M_{1}}{b_{2}} \exp \left[\left(-a_{2}+c_{2} M_{1}\right) \tau_{2}+\exp \left(-\left(-a_{2}+c_{2} M_{1}\right) \tau_{2}\right)-1\right]=M_{2} .
$$

The proof is complete.

The following lemma is a corollary of [30, Theorem 2.1].

Lemma 3.3. Assume that (A1) holds and let $\left(N_{1}(t), N_{2}(t)\right)$ be the solution of (1.1) and (1.2). Then

$$
0<\liminf _{t \rightarrow \infty} N_{i}(t) \leqslant \limsup _{t \rightarrow \infty} N_{i}(t)<\infty, \quad i=1,2
$$

\section{Proofs of the main results}

Proof of Theorem 2.1. By the transformation

$$
x_{i}(t)=N_{i}(t)-N_{i}^{*}, \quad i=1,2,
$$

system (1.10) becomes

$$
\left.\begin{array}{l}
\dot{x}_{1}(t)=\left(N_{1}^{*}+x_{1}(t)\right)\left[-b_{1} x_{1}(t-\tau)-c_{1} x_{2}\left(t-\sigma_{1}\right)\right] \\
\dot{x}_{2}(t)=\left(N_{2}^{*}+x_{2}(t)\right)\left[c_{2} x_{1}\left(t-\sigma_{2}\right)-b_{2} x_{2}(t)\right] .
\end{array}\right\}
$$

Clearly, the global attractivity of $N^{*}$ of system $(1.10)$ is equivalent to that of $(0,0)$ for (4.1), meaning that

$$
\lim _{t \rightarrow \infty} x_{i}(t)=0, \quad i=1,2,
$$

for all solutions $x(t)=\left(x_{1}(t), x_{2}(t)\right)$ with $x_{1}(t)>-N_{1}^{*}$ and $x_{2}(t)>-N_{2}^{*}$ for $t \geqslant 0$. We have two cases to consider in order to prove (4.2).

Case 1. $b_{1} x_{1}(t-\tau)+c_{1} x_{2}\left(t-\sigma_{1}\right)$ or $c_{2} x_{1}\left(t-\sigma_{2}\right)-b_{2} x_{2}(t)$ is non-oscillatory. It is harmless to assume that $b_{1} x_{1}(t-\tau)+c_{1} x_{2}\left(t-\sigma_{1}\right)$ is non-oscillatory. Then, $\dot{x}_{1}(t)$ is signdefinite eventually, which implies that $x_{1}(t)$ is monotonous eventually. By Lemma 3.3, we have $x_{1}(t) \rightarrow \alpha_{1}$ as $t \rightarrow \infty$ and $N_{1}^{*}+\alpha_{1}>0$. On the other hand, using the boundedness of $x_{1}(t)$ and $x_{2}(t)$, we can conclude from (4.1) that both $\dot{x}_{1}(t)$ and $\dot{x}_{2}(t)$ are also bounded on $[0, \infty)$, which implies that $x_{1}(t)$ and $x_{2}(t)$ are uniformly continuous on $[0, \infty)$. It follows 
immediately that $\dot{x}_{1}(t)$ and $\dot{x}_{2}(t)$ are also uniformly continuous on $[0, \infty)$. Therefore, by [4, Lemma 1.2.3], $\dot{x}_{1}(t) \rightarrow 0$ as $t \rightarrow \infty$. Hence, from (4.1), we obtain

$$
b_{1} \alpha_{1}+c_{1} x_{2}\left(t-\sigma_{1}\right) \rightarrow 0 \text { as } t \rightarrow \infty,
$$

which implies that the limit $\alpha_{2}=\lim _{t \rightarrow \infty} x_{2}(t)$ exists. Analogously to the above proof, we have $\dot{x}_{2}(t) \rightarrow 0$ as $t \rightarrow \infty$. Hence, from (4.1) and Lemma 3.3, we have

$$
b_{1} \alpha_{1}+c_{1} \alpha_{2}=0, \quad c_{2} \alpha_{1}-b_{2} \alpha_{2}=0,
$$

which imply that $\alpha_{1}=\alpha_{2}=0$, i.e. (4.2) holds.

Case 2. Both $b_{1} x_{1}(t-\tau)+c_{1} x_{2}\left(t-\sigma_{1}\right)$ and $c_{2} x_{1}\left(t-\sigma_{2}\right)-b_{2} x_{2}(t)$ are oscillatory. Then there exist two infinity sequences $\left\{s_{n}\right\}$ and $\left\{t_{n}\right\}$ such that

$$
\begin{gathered}
b_{1} x_{1}\left(s_{n}-\tau\right)+c_{1} x_{2}\left(s_{n}-\sigma_{1}\right)=0, \quad c_{2} x_{1}\left(t_{n}-\sigma_{2}\right)-b_{2} x_{2}\left(t_{n}\right)=0, \quad n=1,2, \ldots, \\
x_{2}\left(t_{2 n-1}\right) \leqslant x_{2}(t) \leqslant x_{2}\left(t_{2 n}\right) \quad \text { for } t_{2 n-1} \leqslant t \leqslant t_{2 n}, n=1,2, \ldots
\end{gathered}
$$

and

$$
\liminf _{n \rightarrow \infty} x_{2}\left(t_{2 n-1}\right)=\liminf _{t \rightarrow \infty} x_{2}(t) \leqslant \limsup _{t \rightarrow \infty} x_{2}(t)=\limsup _{n \rightarrow \infty} x_{2}\left(t_{2 n}\right) .
$$

Set

$$
-v=\liminf _{t \rightarrow \infty} x_{1}(t) \quad \text { and } \quad u=\limsup _{t \rightarrow \infty} x_{1}(t) .
$$

Then from (4.3)-(4.5), we have

$$
\limsup _{t \rightarrow \infty} x_{2}(t)=\limsup _{n \rightarrow \infty} x\left(t_{2 n}\right)=\frac{c_{2}}{b_{2}} \limsup _{n \rightarrow \infty} x_{1}\left(t_{2 n}-\sigma_{2}\right) \leqslant \frac{c_{2}}{b_{2}} u
$$

and

$$
\liminf _{t \rightarrow \infty} x_{2}(t)=\liminf _{n \rightarrow \infty} x\left(t_{2 n-1}\right)=\frac{c_{2}}{b_{2}} \liminf _{n \rightarrow \infty} x_{1}\left(t_{2 n-1}-\sigma_{2}\right) \geqslant-\frac{c_{2}}{b_{2}} v .
$$

Hence,

$$
0=\lim _{n \rightarrow \infty}\left[b_{1} x_{1}\left(s_{n}-\tau\right)+c_{1} x_{2}\left(s_{n}-\sigma_{1}\right)\right] \leqslant b_{1} u+c_{1} \limsup _{t \rightarrow \infty} x_{2}(t) \leqslant\left(b_{1}+\frac{c_{1} c_{2}}{b_{2}}\right) u
$$

and

$$
0=\lim _{n \rightarrow \infty}\left[b_{1} x_{1}\left(s_{n}-\tau\right)+c_{1} x_{2}\left(s_{n}-\sigma_{1}\right)\right] \geqslant-b_{1} v+c_{1} \liminf _{t \rightarrow \infty} x_{2}(t) \geqslant-\left(b_{1}+\frac{c_{1} c_{2}}{b_{2}}\right) v .
$$

Thus, in view of Lemma 3.3 and the above results, we have

$$
-N_{1}^{*}<-v \leqslant 0 \leqslant u<\infty .
$$

Set $\mu=c_{1} c_{2} / b_{1} b_{2}$. Then $0<\mu<1$. In what follows, we show that $v$ and $u$ satisfy the inequalities

$$
N_{1}^{*}+u \leqslant\left(N_{1}^{*}+\mu v\right) \exp \left[\frac{1-\mu}{N_{1}^{*}} v-\frac{(1-\mu)^{2}(1+2 \mu)}{6 N_{1}^{* 2}(1+\mu)} v^{2}\right]
$$


and

$$
N_{1}^{*}-v \geqslant\left(N_{1}^{*}-\mu u\right) \exp \left[-\frac{1-\mu}{N_{1}^{*}} u-\frac{(1-\mu)^{2}(1+2 \mu)}{6 N_{1}^{* 2}(1+\mu)} u^{2}\right] .
$$

For the sake of simplicity, we set

$$
A=\frac{3(1-\mu)}{2 N_{1}^{*}(1+\mu)}+\frac{\mu(1-\mu)}{N_{1}^{*}(1+\mu)^{2}}=\frac{(1-\mu)(3+5 \mu)}{2 N_{1}^{*}(1+\mu)^{2}} .
$$

Then (2.1) implies $b_{1} \tau \leqslant A$. Let $\varepsilon>0$ be sufficiently small such that $v_{1} \equiv v+\varepsilon<N_{1}^{*}$. Choose $T>0$ such that

$$
-v_{1}<x_{1}(t)<u+\varepsilon \equiv u_{1} \quad \text { and } \quad-\frac{c_{2}}{b_{2}} v_{1}<x_{2}(t)<\frac{c_{2}}{b_{2}} u_{1}, \quad t \geqslant T-\Delta .
$$

Set $v_{2}=(1+\mu) v_{1}$ and $u_{2}=(1+\mu) u_{1}$. Then, from (4.1), we have

$$
\frac{\dot{x}_{1}(t)}{N_{1}^{*}+x_{1}(t)} \leqslant b_{1}\left[-x_{1}(t-\tau)+\mu v_{1}\right] \leqslant b_{1} v_{2}, \quad t \geqslant T
$$

and

$$
-\frac{\dot{x}_{1}(t)}{N_{1}^{*}+x_{1}(t)} \leqslant b_{1}\left[x_{1}(t-\tau)+\mu u_{1}\right] \leqslant b_{1} u_{2}, \quad t \geqslant T .
$$

First, we prove that (4.9) holds. If $u \leqslant \mu v$, then (4.9) obviously holds. Therefore, we will prove (4.9) only in the case when $u>\mu v$. For simplicity, it is harmless to assume that $u>\mu v_{1}$. Thus, we cannot have $x_{1}(t) \leqslant \mu v_{1}$ eventually. On the other hand, if $x_{1}(t) \geqslant \mu v_{1}$ eventually, then it follows from the first inequality in (4.12) that $x_{1}(t)$ is non-increasing and that $u=\lim _{t \rightarrow \infty} x_{1}(t)=\mu v_{1}$. This is also impossible. Therefore, it follows that $x_{1}(t)$ oscillates about $\mu v_{1}$.

Let $\left\{p_{n}\right\}$ be an increasing sequence such that $p_{n} \geqslant T+\Delta, \dot{x}_{1}\left(p_{n}\right)=0, x_{1}\left(p_{n}\right) \geqslant \mu v_{1}$, $\lim _{n \rightarrow \infty} p_{n}=\infty$ and $\lim _{n \rightarrow \infty} x_{1}\left(p_{n}\right)=u$. By (4.12), there exists $\xi_{n} \in\left[p_{n}-\tau, p_{n}\right]$ such that $x_{1}\left(\xi_{n}\right)=\mu v_{1}$. For $t \in\left[\xi_{n}, p_{n}\right]$, integrating (4.12) from $t-\tau$ to $\xi_{n}$ we get

$$
-\ln \frac{N_{1}^{*}+x_{1}(t-\tau)}{N_{1}^{*}+x_{1}\left(\xi_{n}\right)} \leqslant b_{1} v_{2}\left(\xi_{n}+\tau-t\right), \quad \xi_{n} \leqslant t \leqslant p_{n}
$$

Thus,

$$
x_{1}(t-\tau) \geqslant-N_{1}^{*}+\left(N_{1}^{*}+\mu v_{1}\right) \exp \left[-b_{1} v_{2}\left(\xi_{n}+\tau-t\right)\right], \quad \xi_{n} \leqslant t \leqslant p_{n} .
$$

Substituting this into the first inequality in (4.12), we obtain

$$
\frac{\dot{x}_{1}(t)}{N_{1}^{*}+x_{1}(t)} \leqslant\left(N_{1}^{*}+\mu v_{1}\right) b_{1}\left[1-\exp \left(-b_{1} v_{2}\left(\xi_{n}+\tau-t\right)\right)\right], \quad \xi_{n} \leqslant t \leqslant p_{n} .
$$

Combining this with (4.12), we have

$$
\frac{\dot{x}_{1}(t)}{N_{1}^{*}+x_{1}(t)} \leqslant \min \left\{b_{1} v_{2},\left(N_{1}^{*}+\mu v_{1}\right) b_{1}\left[1-\exp \left(-b_{1} v_{2}\left(\xi_{n}+\tau-t\right)\right)\right]\right\}, \quad \xi_{n} \leqslant t \leqslant p_{n} .
$$

Analogously to the proof in $[\mathbf{2 9}]$, we can prove (4.9) by (4.14) and the fact that $b_{1} \tau \leqslant A$. 
Next, we will prove that (4.10) holds as well. If $v=0$, then (4.10) holds naturally. In what follows, we assume that $v>0$. Then, from (4.9), we have

$$
\left.\begin{array}{rl}
u & <N_{1}^{*}(1+\mu) \mathrm{e}^{1-\mu}-N_{1}^{*}<2 N_{1}^{*}, \\
\mu u & <\mu\left[\left(N_{1}^{*}+\mu v\right) \exp \left(\frac{(1-\mu) v}{N_{1}^{*}}\right)-N_{1}^{*}\right]<v<N_{1}^{*} .
\end{array}\right\}
$$

Thus we may assume, without loss of generality, that $v>\mu u_{1}$. In view of this and (4.13), we can show that neither $x_{1}(t) \geqslant-\mu u_{1}$ eventually nor $x_{1}(t) \leqslant-\mu u_{1}$ eventually. Therefore, $x_{1}(t)$ oscillates about $-\mu u_{1}$.

Let $\left\{q_{n}\right\}$ be an increasing sequence such that $q_{n} \geqslant T+\Delta, \dot{x}_{1}\left(q_{n}\right)=0, x_{1}\left(q_{n}\right) \leqslant-\mu u_{1}$, $\lim _{n \rightarrow \infty} q_{n}=\infty$ and $\lim _{n \rightarrow \infty} x_{1}\left(q_{n}\right)=-v$. By (4.13), there exists $\eta_{n} \in\left[q_{n}-\tau, q_{n}\right]$ such that $x_{1}\left(\eta_{n}\right)=-\mu u_{1}$. For $t \in\left[\eta_{n}, q_{n}\right]$, by (4.13), we have

$$
x_{1}(t-\tau) \leqslant\left(N_{1}^{*}-\mu u_{1}\right) \exp \left[b_{1} u_{2}\left(\eta_{n}+\tau-t\right)\right]-N_{1}^{*}, \quad \eta_{n} \leqslant t \leqslant q_{n} .
$$

Substituting this into the first inequality in (4.13), we obtain

$$
-\frac{\dot{x}_{1}(t)}{N_{1}^{*}+x_{1}(t)} \leqslant\left(N_{1}^{*}-\mu u_{1}\right) b_{1}\left[\exp \left(b_{1} u_{2}\left(\eta_{n}+\tau-t\right)\right)-1\right], \quad \eta_{n} \leqslant t \leqslant q_{n} .
$$

Combining this with (4.13), we have

$$
-\frac{\dot{x}_{1}(t)}{N_{1}^{*}+x_{1}(t)} \leqslant \min \left\{b_{1} u_{2},\left(N_{1}^{*}-\mu u_{1}\right) b_{1}\left[\exp \left(b_{1} u_{2}\left(\eta_{n}+\tau-t\right)\right)-1\right]\right\}, \quad \eta_{n} \leqslant t \leqslant q_{n} .
$$

Analogously to the proof in [29], we can prove (4.10) by (4.16) and the fact that $b_{1} \tau \leqslant A$. In view of Lemma 3.1, it follows from (4.9) and (4.10) that $u=v=0$. Thus, (4.2) holds. The proof is complete.

Proof of Theorem 2.2. By the transformation

$$
x_{i}(t)=N_{i}(t)-N_{i}^{*}, \quad i=1,2,
$$

system (1.1) becomes

$$
\left.\begin{array}{l}
\dot{x}_{1}(t)=\left(N_{1}^{*}+x_{1}(t)\right)\left[-b_{1} x_{1}\left(t-\tau_{1}\right)-c_{1} x_{2}\left(t-\sigma_{1}\right)\right], \\
\dot{x}_{2}(t)=\left(N_{2}^{*}+x_{2}(t)\right)\left[c_{2} x_{1}\left(t-\sigma_{2}\right)-b_{2} x_{2}\left(t-\tau_{2}\right)\right] .
\end{array}\right\}
$$

Let $\left(x_{1}(t), x_{2}(t)\right)$ be any solution of (4.17) with $N_{i}^{*}+x_{i}(t)>0$ for $t \geqslant 0$ and $i=1,2$. By Lemma 3.2, there exists $T>0$ such that

$$
N_{i}^{*}+x_{i}(t) \leqslant M_{i}, \quad t \geqslant T, i=1,2 .
$$

We have two cases to consider in order to prove (4.2).

Case 1. $b_{1} x_{1}\left(t-\tau_{1}\right)+c_{1} x_{2}\left(t-\sigma_{1}\right)$ or $c_{2} x_{1}\left(t-\sigma_{2}\right)-b_{2} x_{2}\left(t-\tau_{2}\right)$ is non-oscillatory. In this case, by a similar proof to that of case 1 in Theorem 2.1, we can show that (4.2) holds. 
Case 2. Both $b_{1} x_{1}\left(t-\tau_{1}\right)+c_{1} x_{2}\left(t-\sigma_{1}\right)$ and $c_{2} x_{1}\left(t-\sigma_{2}\right)-b_{2} x_{2}\left(t-\tau_{2}\right)$ are oscillatory. Set

$$
U_{i}=\limsup _{t \rightarrow \infty}\left|x_{i}(t)\right|, \quad i=1,2 .
$$

By Lemma 3.2, $0 \leqslant U_{i}<\infty, i=1,2, \ldots, n$. It suffices to prove that $U_{1}=U_{2}=0$. To this end, assume that $U_{1}>0$ and $U_{2}>0$. Hence, by (4.17), for any given sufficiently small $\varepsilon>0$, there exist two sequences $\left\{t_{i n}\right\}, i=1,2$ with $t_{\text {in }}-\Delta>T$ such that

$$
\left.\begin{array}{rl}
t_{\text {in }} \rightarrow \infty, \quad\left|x_{i}\left(t_{i n}\right)\right| \rightarrow U_{i} \quad \text { as } n \rightarrow \infty, \quad\left|x_{i}\left(t_{i n}\right)\right|>U_{i}-\varepsilon, \\
\left|\dot{x}_{i}\left(t_{i n}\right)\right|=0, \quad\left|x_{i}(t)\right|<U_{i}+\varepsilon \quad \text { for } t \geqslant t_{1},
\end{array}\right\} \quad i=1,2 .
$$

where $t_{1}=\min \left\{t_{i 1}: i=1,2\right\}$. We can assume that $\left|x_{i}\left(t_{i n}\right)\right|=x_{i}\left(t_{i n}\right)$ (if necessary, we use $-x_{i}(t)$ instead of $x_{i}(t)$ and $-b_{i},-c_{i}$ instead of $b_{i}, c_{i}$ for $\left.i=1,2\right)$. Then, by (4.17), we have $0=b_{1} x_{1}\left(t_{1 n}-\tau_{1}\right)+c_{1} x_{2}\left(t_{1 n}-\sigma_{1}\right)$, which yields

$$
x_{1}\left(t_{1 n}-\tau_{1}\right) \leqslant \frac{c_{1}}{b_{1}}\left(U_{2}+\varepsilon\right) \equiv \beta_{1} .
$$

Set

$$
b_{12}= \begin{cases}\frac{\left[2+\left(M_{1} b_{1} \tau_{1}\right)^{2}\right] c_{1}}{\left[2-\left(M_{1} b_{1} \tau_{1}\right)^{2}\right] b_{1}} & \text { if } M_{1} b_{1} \tau_{1} \leqslant 1 \\ \frac{\left[1+2\left(M_{1} b_{1} \tau_{1}\right)\right] c_{1}}{\left[3-2\left(M_{1} b_{1} \tau_{1}\right)\right] b_{1}} & \text { if } M_{1} b_{1} \tau_{1}>1\end{cases}
$$

and

$$
b_{21}= \begin{cases}\frac{\left[2+\left(M_{2} b_{2} \tau_{2}\right)^{2}\right] c_{2}}{\left[2-\left(M_{2} b_{2} \tau_{2}\right)^{2}\right] b_{2}} & \text { if } M_{2} b_{2} \tau_{2} \leqslant 1 \\ \frac{\left[1+2\left(M_{2} b_{2} \tau_{2}\right)\right] c_{2}}{\left[3-2\left(M_{2} b_{2} \tau_{2}\right)\right] b_{2}} & \text { if } M_{2} b_{2} \tau_{2}>1\end{cases}
$$

Then, by $(2.5), b_{12} b_{21}<1$. In what follows, we show that

$$
x_{1}\left(t_{1 n}\right) \leqslant b_{12}\left(U_{2}+\varepsilon\right)+ \begin{cases}\frac{2 \varepsilon\left(M_{1} b_{1} \tau_{1}\right)^{2}}{\left[2-\left(M_{1} b_{1} \tau_{1}\right)^{2}\right]} & \text { if } M_{1} b_{1} \tau_{1} \leqslant 1, \\ \frac{2 \varepsilon\left(2 M_{1} b_{1} \tau_{1}-1\right)}{3-2 M_{1} b_{1} \tau_{1}} & \text { if } M_{1} b_{1} \tau_{1}>1 .\end{cases}
$$

If $x_{1}\left(t_{1 n}\right) \leqslant \beta_{1}$, then (4.20) obviously holds. If $x_{1}\left(t_{1 n}\right)>\beta_{1}$, then there exists $\xi_{1 n} \in$ $\left[t_{1 n}-\tau_{1}, t_{1 n}\right]$ such that $x_{1}\left(\xi_{1 n}\right)=\beta_{1}$. From (4.17) we have

$$
\begin{aligned}
\dot{x}_{1}(t) & \leqslant\left(N_{1}^{*}+x_{1}(t)\right) b_{1}\left[-x_{1}\left(t-\tau_{1}\right)+\beta_{1}\right] \\
& \leqslant M_{1} b_{1}\left[\left(U_{1}+\varepsilon\right)+\beta_{1}\right], \quad t \geqslant T_{2}=t_{1}+\Delta .
\end{aligned}
$$

By (4.21), we have

$$
\beta_{1}-x_{1}\left(t-\tau_{1}\right) \leqslant M_{1} b_{1}\left[\left(U_{1}+\varepsilon\right)+\beta_{1}\right]\left(\xi_{1 n}+\tau_{1}-t\right), \quad \xi_{1 n} \leqslant t \leqslant t_{1 n} .
$$


Substituting this into the first inequality in (4.21), we obtain

$$
\dot{x}_{1}(t) \leqslant\left(M_{1} b_{1}\right)^{2}\left[\left(U_{1}+\varepsilon\right)+\beta_{1}\right]\left(\xi_{1 n}+\tau_{1}-t\right), \quad \xi_{1 n} \leqslant t \leqslant t_{1 n} .
$$

Combining this and (4.21), we have

$$
\dot{x}_{1}(t) \leqslant M_{1} b_{1}\left[\left(U_{1}+\varepsilon\right)+\beta_{1}\right] \min \left\{1, M_{1} b_{1}\left(\xi_{1 n}+\tau_{1}-t\right),\right\}, \quad \xi_{1 n} \leqslant t \leqslant t_{1 n} .
$$

We consider the following three subcases.

Case 2.1. $M_{1} b_{1} \tau_{1} \leqslant 1$. In this case, by (4.23) we have

$$
\begin{aligned}
x_{1}\left(t_{1 n}\right)-x_{1}\left(\xi_{1 n}\right) & \leqslant\left[\left(U_{1}+\varepsilon\right)+\beta_{1}\right]\left(M_{1} b_{1}\right)^{2} \int_{\xi_{1 n}}^{t_{1 n}}\left(\xi_{1 n}+\tau_{1}-t\right) \mathrm{d} t \\
& =\left[\left(U_{1}+\varepsilon\right)+\beta_{1}\right]\left(M_{1} b_{1}\right)^{2}\left[\tau_{1}\left(t_{1 n}-\xi_{1 n}\right)-\frac{1}{2}\left(t_{1 n}-\xi_{1 n}\right)^{2}\right] \\
& \leqslant \frac{1}{2}\left(M_{1} b_{1} \tau_{1}\right)^{2}\left[\left(U_{1}+\varepsilon\right)+\beta_{1}\right] \\
& \leqslant \frac{1}{2}\left(M_{1} b_{1} \tau_{1}\right)^{2}\left[x_{1}\left(t_{1 n}\right)+\beta_{1}+2 \varepsilon\right] .
\end{aligned}
$$

Case 2.2. $M_{1} b_{1} \tau_{1}>1$ and $M_{1} b_{1}\left(t_{1 n}-\xi_{1 n}\right) \leqslant 1$. In this case, by (4.23) we have

$$
\begin{aligned}
x_{1}\left(t_{1 n}\right)-x_{1}\left(\xi_{1 n}\right) & \leqslant\left[\left(U_{1}+\varepsilon\right)+\beta_{1}\right]\left(M_{1} b_{1}\right)^{2} \int_{\xi_{1 n}}^{t_{1 n}}\left(\xi_{1 n}+\tau_{1}-t\right) \mathrm{d} t \\
& =\left[\left(U_{1}+\varepsilon\right)+\beta_{1}\right]\left(M_{1} b_{1}\right)^{2}\left[\tau_{1}\left(t_{1 n}-\xi_{1 n}\right)-\frac{1}{2}\left(t_{1 n}-\xi_{1 n}\right)^{2}\right] \\
& \leqslant \frac{1}{2}\left(2 M_{1} b_{1} \tau_{1}-1\right)\left[\left(U_{1}+\varepsilon\right)+\beta_{1}\right] \\
& \leqslant \frac{1}{2}\left(2 M_{1} b_{1} \tau_{1}-1\right)\left[x_{1}\left(t_{1 n}\right)+\beta_{1}+2 \varepsilon\right] .
\end{aligned}
$$

Case 2.3. $M_{1} b_{1} \tau_{1}>1$ and $M_{1} b_{1}\left(t_{1 n}-\xi_{1 n}\right)>1$. In this case, let $\eta_{1 n} \in\left[\xi_{1 n}, t_{1 n}\right]$ be such that $M_{1} b_{1}\left(t_{1 n}-\eta_{1 n}\right)=1$. Then by (4.23) we have

$$
\begin{aligned}
x_{1}\left(t_{1 n}\right)-x_{1}\left(\xi_{1 n}\right) & \leqslant\left[\left(U_{1}+\varepsilon\right)+\beta_{1}\right] M_{1} b_{1}\left[\eta_{1 n}-\xi_{1 n}+M_{1} b_{1} \int_{\eta_{1 n}}^{t_{1 n}}\left(\xi_{1 n}+\tau_{1}-t\right) \mathrm{d} t\right] \\
& =\left[\left(U_{1}+\varepsilon\right)+\beta_{1}\right]\left[\left(M_{1} b_{1}\right)^{2} \tau_{1}\left(t_{1 n}-\eta_{1 n}\right)-\frac{1}{2}\left(M_{1} b_{1}\right)^{2}\left(t_{1 n}-\eta_{1 n}\right)^{2}\right] \\
& =\frac{1}{2}\left(2 M_{1} b_{1} \tau_{1}-1\right)\left[\left(U_{1}+\varepsilon\right)+\beta_{1}\right] \\
& \leqslant \frac{1}{2}\left(2 M_{1} b_{1} \tau_{1}-1\right)\left[x_{1}\left(t_{1 n}\right)+\beta_{1}+2 \varepsilon\right] .
\end{aligned}
$$

Combining Cases 2.1-2.3, we have

$$
x_{1}\left(t_{1 n}\right) \leqslant \begin{cases}\frac{\left[2+\left(M_{1} b_{1} \tau_{1}\right)^{2}\right] c_{1}}{\left[2-\left(M_{1} b_{1} \tau_{1}\right)^{2}\right] b_{1}}\left(U_{2}+\varepsilon\right)+\frac{2 \varepsilon\left(M_{1} b_{1} \tau_{1}\right)^{2}}{2-\left(M_{1} b_{1} \tau_{1}\right)^{2}} & \text { if } M_{1} b_{1} \tau_{1} \leqslant 1, \\ \frac{\left[1+2\left(M_{1} b_{1} \tau_{1}\right)\right] c_{1}}{\left[3-2\left(M_{1} b_{1} \tau_{1}\right)\right] b_{1}}\left(U_{2}+\varepsilon\right)+\frac{2 \varepsilon\left(2 M_{1} b_{1} \tau_{1}-1\right)}{3-2\left(M_{1} b_{1} \tau_{1}\right)} & \text { if } M_{1} b_{1} \tau_{1}>1 .\end{cases}
$$

This shows that (4.21) is true. Letting $n \rightarrow \infty$ and $\varepsilon \rightarrow 0$ in (4.21), we obtain

$$
U_{1} \leqslant b_{12} U_{2}
$$


Similarly, we have

$$
U_{2} \leqslant b_{21} U_{1}
$$

By (4.23) and (4.24), we have

$$
U_{1} \leqslant b_{12} b_{21} U_{1}<U_{1} \text { and } U_{2} \leqslant b_{12} b_{21} U_{2}<U_{2} .
$$

This is a contradiction. The proof is complete.

Acknowledgements. X.H.T. was supported by a grant of the NNSF China (no. 10771215). X.Z was supported by the NSERC and NCE-MITAC of Canada and by the PREA of Ontario Government. The authors are grateful to the referee for valuable comments which have led to a significant improvement of the presentation of the manuscript.

\section{References}

1. D. I. BARneA, A method and new result for stability and instability of autonomous functional differential equations, SIAM J. Appl. Math. 17 (1969), 681-697.

2. C. Castillo-Chavez and H. R. Thieme, Asymptotically autonomous epidemic models, in Mathematical population dynamics: analysis of heterogeneity, Volume I, Theory of epidemics, pp. 33-50 (Wuerz, Winnipeg, Canada, 1995).

3. K. Gopalsamy, Stability criteria for a linear system $\dot{X}(t)+A(t) X(t-\tau)=0$ and an application to a nonlinear system, Int. J. Sys. Sci. 21 (1990), 1841-1853.

4. K. Gopalsamy, Stability and oscillations in delay-differential equations of population dynamics (Kluwer, Dordrecht, 1992).

5. K. Gopalsamy and X. He, Global stability in $n$-species competition modelled by puredelay type systems, II, Non-autonomous case, Can. Appl. Math. Q. 6 (1998), 17-43.

6. X. He, Stability and delays in a predator-prey system, J. Math. Analysis Applic. 198 (1996), 355-370.

7. X. HE, The Liapunov functionals for Lotka-Volterra type models, SIAM J. Appl. Math. 58 (1998), 1222-1236.

8. X. He, Global stability in non-autonomous Lotka-Volterra systems of 'pure-delay type', Diff. Integ. Eqns 11 (1998), 293-310.

9. J. Hofbauer And J. W.-H. So, Diagonal dominance and harmless off-diagonal delays, Proc. Am. Math. Soc. 128 (2000), 2675-2682.

10. A. Ivanov, E. Liz And S. Trofimchuk, Halanay inequality, Yorke $3 / 2$ stability criterion, and differential equations with maxima, Tohoku Math. J. 54 (2002), 277-295.

11. T. KRiszTin, On stability properties for one-dimensional functional-differential equations, Funkcial. Ekvac. 34 (1991), 241-256.

12. Y. KUANG, Delay-differential equations with applications in population dynamics (Academic, Boston, MA, 1993).

13. Y. KUANG, Global stability in delay-differential systems without dominating instantaneous negative feedbacks, J. Diff. Eqns 119 (1995), 503-532.

14. Y. KuAng AND H. L. Smith, Convergence in Lotka-Volterra type diffusive delay systems without dominating instantaneous negative feedbacks, J. Austral. Math. Soc. B 34 (1993), 471-493.

15. Y. KuAng And H. L. Smith, Convergence in Lotka-Volterra-type diffusive delay systems without instantaneous feedbacks, Proc. R. Soc. Edinb. A 123 (1993), 45-58.

16. X. Li, Z. C. WANG AND X. Zou, A $3 / 2$ stability result for the logistic equation with two delays, Commun. Appl. Analysis 7 (2003), 193-202. 
17. E. Liz, M. Pinto, G. Robledo and S. Trofimchuk, Wright-type delay-differential equations with negative Schwarzian, Discrete Contin. Dynam. Syst. B 9 (2003), 309-321.

18. A. D. Myshkis, Linear differential equations with retarded arguments (Nauka, Moscow, 1972).

19. H. L. SMith, Monotone dynamical systems: an introduction to the theory of competitive and cooperative systems (American Mathematical Society, Providence, RI, 1995).

20. J. W.-H. So AND J. S. YU, Global attractivity for a population model with time delay, Proc. Am. Math. Soc. 123 (1995), 2687-2694.

21. J. W.-H. So AND J. S. YU, On the uniform stability for a 'food-limited' population model with time delay, Proc. R. Soc. Edinb. A 125 (1995), 991-1002.

22. J. W.-H. So AND J. S. YU, Global stability for a general population model with time delays, Fields Inst. Commun. 21 (1999), 447-457.

23. J. W.-H. So, X. H. TANG AND X. ZOU, Stability in linear delay systems without instantaneous negative feedback, SIAM J. Math. Analysis 33 (2002), 1297-1304.

24. X. H. TANG AND J. S. YU, Global existence and global attractivity of solution for a class of nonlinear functional differential equations, Chin. Annals Math. A 21 (2000), 655-666.

25. X. H. TANG AND J. S. YU, Global attractivity of the zero solution of a 'Lotka-Volterra' type functional differential equation, Chin. Annals Math. A 22 (2000), 285-296.

26. X. H. TANG AND J. S. YU, 3/2-global attractivity of the zero solution of the 'food-limited' type functional differential equations, Sci. China A 44 (2001), 610-618.

27. X. H. TANG AND X. Zou, 3/2-type criteria for global attractivity of Lotka-Volterra competition system without instantaneous negative feedbacks, J. Diff. Eqns 186 (2002), 420-439.

28. X. H. TANG AND X. ZOU, A 3/2 stability result for a regulated logistic growth, Discrete Contin. Dynam. Syst. B 2 (2002), 265-278.

29. X. H. TANG AND X. ZOU, Global attractivity of non-autonomous Lotka-Volterra competition system without instantaneous negative feedbacks, J. Diff. Eqns 192 (2003), 502-535.

30. W. WANG And Z. MA, Harmless delays for uniform persistence, J. Math. Analysis Applic. 158 (1991), 256-268.

31. E. M. Wright, A nonlinear difference-differential equation, J. Reine Angew. Math. 194 (1955), 66-87.

32. T. YONEYAMA, On the $3 / 2$ stability theorem for one-dimensional delay-differential equations, J. Math. Analysis Applic. 125 (1987), 161-173.

33. T. Yoneyama, On the $3 / 2$ stability theorem for one-dimensional delay-differential equations with unbounded delay, J. Math. Analysis Applic. 165 (1992), 133-143.

34. J. A. Yorke, Asymptotic stability for one-dimensional differential-delay equations, $J$. Diff. Eqns 7 (1970), 189-202. 